Cahiers d'études africaines

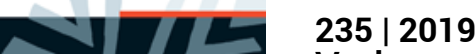

Varia

\title{
« Ruines à rebours »
}

Pour une lecture littéraire des textes ethnographiques de Paul Hazoumé

"Ruines à rebours". For a Literary Reading of Paul Hazoumés Ethnographic

Writings

Ninon Chavoz

\section{OpenEdition}

\section{Journals}

Édition électronique

URL : https://journals.openedition.org/etudesafricaines/26999

DOI : 10.4000/etudesafricaines.26999

ISSN : 1777-5353

Éditeur

Éditions de l'EHESS

\section{Édition imprimée}

Date de publication : 15 septembre 2019

Pagination : 815-838

ISSN : 0008-0055

\section{Référence électronique}

Ninon Chavoz, " «Ruines à rebours » », Cahiers d'études africaines [En ligne], 235 | 2019, mis en ligne le 01 janvier 2022, consulté le 07 janvier 2022. URL : http://journals.openedition.org/etudesafricaines/ 26999 ; DOI : https://doi.org/10.4000/etudesafricaines.26999 


\title{
«Ruines à rebours»
}

\author{
Pour une lecture littéraire \\ des textes ethnographiques de Paul Hazoumé
}

«La porte de l'école est un concept qui devrait faire réfléchir les architectes et les grammairiens » (Hocquart 2010 : 35).

Franchir la «porte de l'école» — et plus tard celle de l'institution universitaire - a assurément constitué une étape décisive dans la destinée de l'écrivain dahoméen Paul Hazoumé (1890-1980). Descendant d'une famille de dignitaires de la cour du roi Sodji de Porto-Novo ${ }^{1}$, brillant diplômé de l'École William Ponty de Saint-Louis, il se distingue comme l'un des meilleurs disciples du père Francis Aupiais, membre de la Société des missions africaines qui s'efforça de mettre en place un système éducatif novateur, propice à la création d'une élite dahoméenne ouverte à la connaissance des cultures locales (Balard 1999). C'est donc d'abord en tant qu'élève, puis en tant qu' instituteur, que Paul Hazoumé, pour reprendre les mots de Georges Hardy (1949: 21), «fait prime sur le marché »: il ne cesse, en d'autres termes, d'ouvrir et de franchir la «porte de l'école».

Cette distinction scolaire, appuyée par l'institution religieuse et coloniale, infléchit durablement la réception d'un auteur volontiers rattaché à la qualification ambiguë «d'écrivain instituteur» (Senghor 1947 ; Derive 1980). Le reproche de francophilie souvent adressé à Hazoumé se double dès lors d'une remise en cause de sa pratique littéraire qui, quand elle n'est pas tout bonnement niée, se voit reléguée au rang d'un laborieux exercice scolaire, «plus scientifique en un sens que littéraire » (Senghor 1947). Les conséquences grammaticales du franchissement de la « porte de l'école » en contexte colonial sont ainsi examinées par Albert Gandonou, qui souligne tout ce que l'unique

1. Voir à ce sujet la préface de Jean Cornevin à la seconde édition de Doguicimi (HAZoumé 1972 : III-IV). 
roman de Paul Hazoumé, Doguicimi, a de conservateur en matière de style et de vocabulaire : l'extrême correction de la syntaxe, jamais rétive à l'emploi d'un subjonctif imparfait, va de pair avec un usage minimal du néologisme ou du « régionalisme », compris ici comme l'introduction dans le lexique d'un terme allogène désignant des realia locales (Gandonou 2002).

Notre propos n'est cependant pas de revenir sur ce qualificatif d'écrivaininstituteur pour démontrer en quoi le roman, en dépit d'une forme a priori classique, peut être lu comme un texte audacieux, beaucoup plus émancipé du modèle européen que ne le laisse penser la dénonciation récurrente d'Hazoumé comme écrivain colonial (Coundouriotis 1999). Portant notre regard en amont, nous souhaitons ici revenir sur les textes qui précèdent et, sans doute, préparent le succès de Doguicimi en 1938 : avant cette consécration littéraire, confirmée par l'octroi d'un prix décerné par l'Académie française pour la qualité de la langue, Hazoumé a en effet publié un essai ethnographique, intitulé Le Pacte de sang au Dahomey (1937), ainsi que de nombreux articles, parus pour la plupart dans la revue La Reconnaissance Africaine fondée à Porto Novo par Francis Aupiais. Ces travaux abondants ont joué un rôle crucial dans la carrière de l'écrivain, comme en témoigne la correspondance qu'il entretient avec son mentor, rappelé en métropole à la suite de conflits avec la hiérarchie de son ordre: la publication difficile du Pacte de sang, soumis à l'Institut d'ethnographie de Paris, constitue l'un des sujets les plus fréquemment et les plus longuement abordés dans les lettres que s'échangent les deux hommes. Pourtant, Le Pacte de sang et les articles parus dans La Reconnaissance Africaine n' ont que peu retenu l'attention de la critique : l'ouvrage dirigé par Adrien Huannou et Robert Mane (1987) au sujet de Paul Hazoumé est ainsi essentiellement consacré au commentaire de Doguicimi. Cette occultation relative des écrits ethnographiques est d'autant plus regrettable qu'une lecture croisée des textes hazouméens permet d'établir des continuités thématiques et idéologiques, voire d'étayer une remise en cause de la posture colonialiste et francophile fréquemment imputée à l'auteur. Plus encore, l'analyse de ces textes précoces conduit à éclairer les circonstances d'une « fabrique des savoirs » (Gary-Tounkara \& Nativel 2012) locale et s' inscrit dans la remise en question contemporaine du rôle subalterne et marginal longtemps prêté à l'intermédiaire (Lawrance, Osborn \& Roberts 2006 ; Jézéquel 2007 ; Labrune-Badiane \& Smith 2018). Revenir sur ces écrits, dont le prétexte affiché réside dans la production d'une documentation destinée à « fournir à la colonisation les informations auxquelles elle a droit » (Hazoumé 1937), conduit, par conséquent, à combler un manque et à resituer l'œuvre hazouméenne — régulièrement oubliée par l'histoire littéraire francophone - dans le contexte d'une écriture « régionaliste», nourrie par 
une pratique ethnographique dont le missionnaire Francis Aupiais (2016) se fait le vecteur paradoxal.

En abordant les textes antérieurs à Doguicimi et en les soumettant à un prisme d'interprétation proprement littéraire, nous souhaitons également prendre le contrepied de deux lectures concurrentes imposées à l'œuvre de Paul Hazoumé. La plus courante, largement portée par le discours préfaciel qui accompagne la première édition du roman, tend à cantonner le texte au statut de document ethnographique en lui refusant toute qualité littéraire. En reprenant l'analyse proposée par Laurent Dubreuil (2008) au sujet de Force-Bonté de Bakary Diallo ou du Fils du pauvre de Mouloud Feraoun, on pourrait aller jusqu'à considérer ce déni de littérarité comme une forme de censure voilée, visant à prévenir l'entrée des auteurs francophones dans le champ réservé de la littérature française. Relire les articles ethnographiques de Paul Hazoumé en tant que textes littéraires revient à renverser cette position d'exclusion de l'auteur francophone, en mettant en évidence tout ce qui peut rattacher son écriture - fût-elle a priori considérée comme scientifique - à une pratique littéraire, comprise à la fois comme narration et comme investissement discursif du texte par une subjectivité (Warner 2016) : l'article érudit devient alors le foyer d'un «moi » d'intermédiaire problématique, partagé entre ses origines porto-noviennes et sa formation occidentale.

L'autre lecture à laquelle nous voudrions nous opposer tend au contraire à interpréter la trajectoire de l'auteur francophone comme un parcours téléologique qui aboutirait, au fruit d'un itinéraire plus ou moins heurté, au fantasme romantique du « sacre de l'écrivain » (Bénichou 1973). Cette vision du trajet de l'auteur impose de considérer les écrits scientifiques comme des « laissez-passer », destinés à être éclipsés par le texte littéraire qui en constitue l'unique horizon. Dans le cas de l'écrivain francophone, l'érudition devient ainsi un simple « pré-texte » et le préalable nécessaire à une éventuelle gloire littéraire. Plutôt que de voir en Hazoumé le modèle de ces trajectoires d'ascension linéaire jusqu'au faîte de la reconnaissance littéraire, nous entendons ici placer les écrits de l'auteur sur un pied d'égalité pour mieux examiner leurs zones de porosité. Il semble alors bien plus pertinent de rapprocher Hazoumé du paradigme du double discours, ou du « second livre » élaboré par Vincent Debaene (2010), selon qui l'œuvre littéraire offre aux anthropologues la possibilité d'exprimer le reliquat d'une expérience irréductible à l'étude monographique : on dira alors qu'Hazoumé a écrit le Pacte de sang et Doguicimi, comme Michel Leiris a écrit L'Afrique fantôme (1934) et La Possession et ses aspects théâtraux chez les Éthiopiens de Gondar (1958), ou, pour respecter la succession chronologique de l'essai et du roman, comme Marcel Griaule a écrit Silhouettes et graffiti abyssins (1929) et Les Flambeurs 
d'hommes (1934). Il s'agit donc moins d'établir une gradation entre les œuvres que de penser leurs correspondances et leurs complémentarités.

Lire les écrits ethnographiques de Paul Hazoumé comme des textes littéraires incite par conséquent à refuser leur qualification exclusivement documentaire, et à réfuter simultanément l'hypothèse d'une trajectoire téléologique de l'écrivain francophone. Franchir «la porte de l'école»-ou, en l'occurrence, de l'Institut d'ethnologie de Paris — oblige certes Hazoumé à se plier aux exigences et aux relectures plus ou moins tatillonnes de ses maîtres, mais rien ne permet de voir là l'indice sûr d'un enfermement disciplinaire ou idéologique. Après avoir procédé à un exposé succinct des contextes et des lieux de publication des premiers écrits hazouméens, nous montrerons donc comment ces textes, pourtant considérés comme ethnographiques, ont pu constituer le support de l'invention de personnages romanesques ainsi que la matrice d'une poétique de la nostalgie, pour partie commune aux premiers écrivains-instituteurs (Labrune-Badiane \& Smith 2018). À ce titre, l'emprunt liminaire que nous faisons à l'œuvre d'Emmanuel Hocquart ne tient pas uniquement de la citation heuristique : le rapprochement avec la description de Tanger que propose le poète invite à prendre acte de la capacité de l'écriture hazouméenne à dire poétiquement la transformation d'un Dahomey colonial où fleurissent les monuments et les ruines.

\section{Hazoumé «reconnaissant»: le régionalisme selon Francis Aupiais}

La carrière ethnographique de Paul Hazoumé est intimement liée aux projets qu'il partage avec son mentor, Francis Aupiais : la création d'une revue dahoméenne, La Reconnaissance Africaine, à laquelle sont invités à prendre part les meilleurs élèves des grand et petit séminaires, vise ainsi à mettre en œuvre le projet « régionaliste » que définit Aupiais (1927) à l'occasion de la cinquième semaine de missiologie de Louvain. Dans un contexte où le débat porte sur les enjeux de la constitution d'un clergé africain, ce « régionalisme » politique entend offrir une troisième voie adaptative, distincte de l'assimilation autant que de l'opposition violente à l'influence occidentale. Pour favoriser « une large compréhension de la petite patrie », Aupiais développe ainsi un programme pratique, fondé, entre autres, sur la création d'une revue locale qui a pour mission de diffuser :

[...] les traditions orales concernant l'histoire des villes et des tribus, les grands chants nationaux (guerriers, religieux, funéraires), des spécimens d'un folklore d'ailleurs abondant, des milliers de proverbes qui condensent en des formules lapidaires l'observation, 
la morale, l'esprit, la sagesse de ces populations, bref tout ce qui se rapporte à la religion, au droit, à la politesse, aux coutumes, etc... (Aupiais 1927 : 91).

C'est dans ce cadre que se déploie principalement l'écriture ethnographique de Paul Hazoumé qui, à compter de la création de La Reconnaissance Africaine, se détourne du Bulletin de l'enseignement de l'Afrique Occidentale Française auquel il avait déjà donné quelques articles, et se consacre pleinement au projet initié par Aupiais. En dépit de son assise en apparence strictement locale, la revue constitue une vitrine pour l'élite intellectuelle dahoméenne, à laquelle les campagnes «mélanophiles » (Saulnier 2018: 64) du missionnaire confèrent une certaine visibilité en métropole. Pour Hazoumé, elle fait assurément office de laboratoire d'écriture : non content d'y publier, à l'instigation d'Aupiais, les premiers chapitres du Pacte de sang au Dahomey, il cultive également dès ses premiers articles une attention littéraire à l'économie du texte, manifeste notamment dans le soin qu'il accorde à la conception des personnages.

\section{La Reconnaissance Africaine: sanctuaire et laboratoire}

Revue bimensuelle parue entre août 1935 et mars 1927, La Reconnaissance Africaine se construit à la marge d'un projet architectural : comme le signalent les premiers numéros, la publication est adossée au financement de la construction d'une église à Porto-Novo, et le bulletin doit permettre d'informer les fidèles et les souscripteurs. La Reconnaissance Africaine se conçoit donc d'abord comme un projet local, alimenté par le souci de diffuser la pensée chrétienne dans l'espace dahoméen : la « communauté imaginaire » (Anderson 1996) délimitée par la circulation de l'écrit demeure a priori congrue, cantonnée à un espace géographique et idéologique restreint.

Pourtant, la vocation du monument auquel s'accole La Reconnaissance Africaine ne saurait être comprise au seul prisme du prosélytisme religieux, et il serait faux de la lire comme un bulletin paroissial amélioré. L'église qui, à partir du vingt-troisième numéro, figure en couverture du bulletin, tient en effet également lieu de monument aux morts de la guerre franco-dahoméenne : on a donc affaire à un monument-Janus, à la fois édifice sacré et «lieu de mémoire » historique de la conquête coloniale. La Reconnaissance Africaine, qualifiée en sous-titre d' « Organe d'enseignement religieux et d'études historiques » hérite bel et bien de cette double identité : le bulletin ne se contente pas de relayer l'activité de la communauté chrétienne, mais entend également donner une visibilité aux «études historiques » dahoméennes. Cette double mission conduit Aupiais et les autres contributeurs à rechercher un équilibre 
entre la vocation apologétique et la tentation documentaire : l'examen des sommaires de la revue permet à cet égard de mettre en évidence son glissement progressif vers un régionalisme érudit, qui relègue au second plan le catéchisme et les lettres chrétiennes. Malgré son rattachement au chantier de la nouvelle église de Porto-Novo, La Reconnaissance Africaine gagne progressivement en envergure et tient lieu de vitrine efficace pour les jeunes élites dahoméennes rassemblées par Aupiais. Le premier texte publié par Paul Hazoumé, consacré à l'histoire de l'amazone Tata Ajachè, retient ainsi l'attention de Maurice Delafosse, qui mentionne le nom de l'auteur dans un article de la Dépêche coloniale, paru le 21 avril 1926. Le séjour d'Aupiais en France ne fait que renforcer la visibilité de ces parutions, dont il s'emploie à vanter les mérites auprès des savants de l'Institut d'ethnographie autant qu'auprès de sa hiérarchie ecclésiastique. Deux lettres adressées à Paul Hazoumé témoignent ainsi de l'importance que le missionnaire accorde à la promotion d'un bulletin qui trouve l'essentiel de son lectorat en dehors de la colonie :

Notre petite Reconnaissance si peu connue et appréciée au Dahomey me vaut ici des louanges sans fin. Nous ne nous sommes donc pas trompés, nous pouvons avoir confiance, continuons notre sillon avec courage, les plus beaux espoirs nous sont permis. Désormais la Reconnaissance Africaine est connue, et bien connue, je puis dire de l'Europe entière, parce que j'ai parlé d'elle au poste de radiophonie de la Tour Eiffel, qui, comme tu le sais, est écouté dans des centaines de millions de postes en famille. [...] La Reconnaissance Africaine est entrée dans l'histoire littéraire coloniale (lettre à Paul Hazoumé, datée du 7 février 1927 [Aupiais 2018: 52]).

Quelle joie, quel triomphe, Mon cher Paul, de voir notre chère revue ouverte sur le bureau de sa Sainteté ! Le pape feuilletait et je voyais passer sous ses doigts et sous ses yeux les titres de vos articles!! Comme je leur trouvais un autre sens et une autre beauté, à ces titres! Comme votre travail me paraissait grand dans ce cadre universel de la Ville qui est la tête ou la mère de toutes les nations (lettre à Paul Hazoumé, datée du 2 mai 1928 [Aupiais 2018: 80]).

Évoquant non sans grandiloquence ses interventions à Radio Tour Eiffel et sa rencontre avec le pape Pie XI, Aupiais souligne la vocation du régionalisme dahoméen à acquérir un rayonnement impérial, si ce n'est « universel». Loin de l'écarter des circuits scientifiques agréés par la métropole, l'implication d'Hazoumé dans la conception et dans l'édition de ce bulletin constitue par conséquent un tournant décisif dans sa carrière de chercheur et d'écrivain. Quand bien même les premiers numéros n'auraient été tirés, à en croire les correspondances, qu'à quelques deux cents exemplaires, La Reconnaissance Africaine constitue un vecteur de diffusion efficace pour les textes des auteurs 
dahoméens et œuvre, à ce titre, en faveur de l'inclusion progressive des élites locales dans le champ éditorial métropolitain.

L'engagement de Paul Hazoumé dans le projet porté par Aupiais est indéniable : non content de participer à la production et à la diffusion de la revue, il figure au sommaire de vingt-neuf des quarante-cinq numéros, et contribue ainsi à près de $65 \%$ des publications. La proportion s'élargit encore si on tient compte des cas de double parution — notamment dans les trois derniers numéros du journal, pour lesquels Hazoumé fournit deux séries d'articles parallèles, respectivement consacrées au « Pacte de sang au Dahomey » et au «Calendrier dahoméen ». Au-delà de cette simple évaluation quantitative, le rôle prépondérant confié à Hazoumé se manifeste également dans la forme de ses articles : le jeune auteur bénéficie en effet de la possibilité de publier des textes particulièrement longs, qui s'apparentent à des feuilletons répartis sur une série continue ou discontinue de numéros. Le « Journal de voyage de Cotonou à Dassa-Zoumé » s'étend ainsi sur huit épisodes, entre mars et août 1926. Encore ce récit de voyage reste-t-il interrompu en chemin : la dernière section, publiée au vingt-neuvième numéro du bulletin s'ouvre sur la mention «à suivre », sans que celle-ci soit honorée dans les parutions ultérieures.

Les nombreuses contributions d'Hazoumé se distinguent par une variété thématique qui ne s'explique pas uniquement par la double spécialisation scientifique et religieuse du bulletin. Rares sont en effet les articles d'Hazoumé qui se rattachent exclusivement à la vocation apologétique de la revue : seul un texte du numéro 42, daté de septembre 1927, est dédié à «l'ordination du premier sous-diacre dahoméen à l'église de Ouidah». La plus grande partie des contributions d'Hazoumé abondent en revanche dans la veine des «études historiques » et ethnographiques : en amont des textes traitant du «pacte de sang » et du «calendrier dahoméen » dans les derniers numéros, on citera ainsi plusieurs articles consacrés à la royauté d'Abomey (« Héroïsme d'une amazone dahoméenne », ${ }^{\text {os }} 1,2$ et 3), à la ville de Ouidah ("Aperçu historique sur les origines de Ouidah, ${ }^{\text {os }} 4,5,7,8,10$ et 11), et un ensemble de textes dédiés aux croyances vodun ("L'exécution de la justice dans l'ancien royaume de Porto-Novo », nos 31-32; « Cérémonie expiatoire faite par une personne qui a été consignée chez un fétiche », ${ }^{\text {os }} 34$ et 37 ). On notera enfin qu'Hazoumé est aussi l'auteur, dans le premier numéro du bulletin, d'une «lettre à nos cousins d'Abomey », qui tient autant de l'épître engagée que de l'article scientifique : se targuant de la position intermédiaire que lui confèrent son origine porto-novienne et la tradition francophile de ce protectorat, Hazoumé revient sur le conflit qui a opposé les impérialismes rivaux de la France et du royaume d'Abomey pour recommander l'apaisement, et appeler les vaincus à « apporter leur pierre » au monument de La Reconnaissance Africaine. 
Si le bulletin du père Aupiais prend appui sur la « ruine à rebours » qu'est la construction d'un monument liturgique et commémoratif, il n'en demeure donc pas moins avant tout un laboratoire où l'auteur peut s'essayer à des formes d'écriture variées, tout en proposant une circulation informée dans la mosaïque ethnique et culturelle que constitue alors la colonie du Dahomey.

\section{Le Pacte de sang: un «modèle du genre »}

Les travaux rassemblés dans la revue doivent, aux yeux d'Aupiais, aboutir à la publication de l'essai ethnographique de Paul Hazoumé aux presses de l'Institut d'ethnologie de Paris, où son disciple voisinerait avec des noms aussi prestigieux que ceux de Marcel Griaule, Henri Gaden ou Théodore Monod. Pour le missionnaire, il s'agit non seulement de faire connaître les écrits du meilleur d'entre ses élèves dahoméens, mais aussi de donner une visibilité à son grand projet « régionaliste », et d'infléchir les représentations de l'Afrique en métropole en donnant voix à des textes écrits par des spécialistes locaux : comme il le signale à Hazoumé, le Pacte doit faire figure de « modèle du genre $»^{2}$. Pourtant, la publication de cet essai ethnographique se heurte à de nombreux obstacles, qui tiennent autant à des contraintes circonstancielles qu' aux réticences opposées par l'institution à la publication des écrits d'ethnographes locaux souvent cantonnés au rang d'informateurs anonymes (Aggarwal 1999).

La correspondance entre Hazoumé et Aupiais permet d'établir que le missionnaire se rend en France en 1926 avec le ferme projet de faire publier le manuscrit de son protégé, mais l'entreprise n'aboutit que plus de dix ans plus tard, en 1937. De fait, le décès de Maurice Delafosse, qui constituait le principal soutien métropolitain du régionalisme dahoméen, retarde les perspectives de publication et contraint Aupiais à se constituer un nouveau réseau, en abordant notamment Paul Rivet et Henri Labouret et en fréquentant assidûment les cours de l'Institut. Les réticences de ces nouveaux lecteurs du Pacte ressurgissent à intervalles réguliers dans les correspondances et semblent mettre le projet en péril à plusieurs reprises, tant Hazoumé supporte mal les retards et les interventions de ses relecteurs. Après avoir longuement séjourné entre les mains de Marcel Mauss, le manuscrit est confié en 1934 à Bernard Maupoil chargé, à l'occasion d'un voyage au Dahomey, de discuter

2. «Mon cher Paul, je te prie, je te supplie de laisser ton manuscrit entre les mains de ces Messieurs de l'Institut d'Ethnologie qui lanceront ton livre comme un modèle du genre, ce qui te donnera une consécration d'une valeur unique » (Lettre à Paul Hazoumé, datée du 9 juin 1934 [Aupiais 2018: 115]). 
avec l'auteur des corrections nécessaires. Entretemps, Aupiais a encouragé Hazoumé à publier dans La Reconnaissance Africaine des passages du Pacte, pour mieux en démontrer la qualité et en hâter la publication : des extraits conséquents paraissent ainsi dans les numéros 40 à 45 du bulletin, représentant l'équivalent des trois premiers chapitres de l'essai. En dépit des plaintes répétées d'Hazoumé, qui proteste contre les transformations imposées à son texte, la lecture comparée des deux versions de ces chapitres révèle peu de différences significatives : en dehors de l'ajout massif de notes visant à apporter des précisions historiques ou étymologiques, les modifications exigées sont avant tout cosmétiques et se traduisent essentiellement par des corrections orthographiques — notamment pour les ethnonymes - et par quelques inflexions syntaxiques mineures ${ }^{3}$. On ne saurait donc parler, à tout le moins pour les chapitres en question, d'un processus de réécriture allographe : contrairement à ce qui a pu se produire lors de certains dialogues entre écrivains et éditeurs (King 2002 ; Ducournau 2017), la tonalité générale de l'essai n'est pas modifiée. Plus encore, il est frappant de constater que les passages qui, à en croire les correspondances, suscitent les plus importantes critiques, semblent avoir été maintenus : c'est notamment le cas du chapitre final, intitulé «Essai d'analyse critique », qui inclut une dénonciation des superstitions inhérentes à la conclusion rituelle du «pacte de sang ».

3. On pourra comparer à titre d'exemple les deux passages suivants : «Les Adjas furent longtemps les seuls dépositaires du Pacte de sang qui, à la suite de leurs émigrations seulement, se répandit parmi les autres peuplades. Les premiers «frères » attachaient une grande importance au Pacte sans lequel ils ne pouvaient obtenir des confidences sur certaines affaires du pays, ni espérer une assistance assurée en cas de malheur. La classe des cultivateurs, des guerriers et des chasseurs comptait alors les plus nombreux adeptes de cette pratique » (version 1, parue dans La Reconnaissance africaine, $\mathrm{n}^{\circ} 43$, daté du $1^{\text {er }}$ octobre 1927). «Les Adjanou furent très longtemps les seuls dépositaires des secrets du Pacte de sang qui, à la suite de leurs émigrations seulement, se répandit parmi les autres peuplades. Les premiers « frères » attachaient une grande importance au Pacte sans lequel ils ne pouvaient obtenir des confidences sur certaines affaires du pays, ni compter sur une assistance ferme en cas de malheur. La classe des cultivateurs, des guerriers et des chasseurs comptait alors les plus nombreux adeptes de cette pratique » (version 2, HAzoumé 1937 : 19, nos italiques). On ajoutera que la seconde version se clôt sur une note de bas de page, dont la teneur est la suivante : "Même les villages contractaient le Pacte. Ils en tiraient de grands avantages à cette époque d'insécurité. Les villages qui voulaient se lier de Pacte déléguaient des notables qui se rencontraient dans une forêt limitrophe et procédaient à la cérémonie. Chaque village prenait l'engagement, par la voix de ses représentants, de prévenir son allié des invasions le menaçant à son insu, et, en cas d'attaque d'un ennemi, de se porter au secours de l'allié. La tradition rapporte que le royaume d'Allada prévint chaque fois le royaume Houéda des attaques du royaume d'Abomey et fut pour cela conquis en 1727, après la victoire d'Agadja sur Houfon, roi Houéda. Il est fort probable que le royaume d'Allada n'a fait qu'accomplir un devoir imposé par le Pacte le liant au royaume Houéda. » 
En dépit de difficultés certaines qui retardent de plus de dix ans la publication de l'essai, le projet régionaliste initié avec La Reconnaissance Africaine finit donc par porter ses fruits : la parution de Pacte de sang permet à l'érudit indigène d'entrer dans le champ scientifique métropolitain, sans que la lecture comparée des différentes leçons du texte autorise à parler d'une réécriture forcée.

\section{Tata Ajachè et I'espion, " personnages » ethnographiques}

Le retour sur deux « feuilletons » publiés dans les pages de La Reconnaissance Africaine permet de mettre en évidence le rôle qu'a joué l'expérience régionaliste initiée par Francis Aupiais pour préparer Hazoumé à une publication scientifique agréée par l'institution métropolitaine, mais aussi à une création d'ordre plus littéraire. La notion de " roman ethnographique » (Riesz 2007) incite ainsi à l'examen des porosités entre écriture scientifique et littérarité et conduit à déceler alternativement une tentation littéraire de l'ethnographie et une tentation ethnographique de la littérature. Nous nous arrêterons à ce titre sur la construction de deux personnages — l'amazone et l'espion — qui, s'ils participent indubitablement d'un exposé de l'histoire du Dahomey, se démarquent également, dès leurs premières apparitions sous la plume de Paul Hazoumé, en tant que personnages romanesques.

\section{Monument à l'Amazone}

La première contribution de Paul Hazoumé à La Reconnaissance Africaine est consacrée au récit de sa rencontre avec une amazone - soit avec l'une de ces redoutables guerrières qui ont contribué à forger dans la littérature coloniale la réputation d'un royaume d'Abomey barbare et sanguinaire. La biographie de l'amazone Tata Ajachè Soupo Ma Ha Awouinyan ne saurait cependant être considérée comme l'évocation d'une troupière parmi d'autres : bien plus, c'est l'histoire d'un individu hors du commun qu'Hazoumé s'attache à retracer. Capturée lors de l'une des campagnes militaires du roi Guézo, l'amazone se distingue d'emblée par sa cruauté lors des combats, notamment lorsqu'elle s'attaque à trois paysans nago occupés à labourer leurs champs et tue l'un d'eux d'un coup de houe sur la nuque ${ }^{4}$. Ces exploits — dont Hazoumé ne

4. «Deux des ennemis eurent assez de présence d'esprit pour se sauver et gagner à temps le fourré voisin. Leur compagnon fut comme fiché au sol. En deux bonds je l'atteignis. Ce fut à cet instant qu'il eut conscience du péril ; il voulut fuir. C'était trop tard. J'avais ramassé déjà une houe. Je lui en assenai un grand coup sur la nuque. (Ce que disant, la 
manque pas de souligner l'horreur barbare — lui valent d'attirer l'attention du roi qui, bravant toutes les règles de la cour, fait d'elle sa maîtresse. Lorsque l'amazone tombe enceinte et que les indices de sa grossesse ne peuvent plus être dissimulés, elle est mise au supplice et sommée de révéler le nom du coupable. L'héroïsme qu'elle met à refuser de trahir son maitre ne fait que renforcer l'ardeur amoureuse du prince, qui finit par la prendre publiquement pour épouse et immortalise ses exploits en les faisant représenter sous forme de bas-relief sur les murs du palais (Biton 2000 : planche XII). L'histoire de l'amazone se trouve ainsi intégrée à la geste du royaume: Tata Ajachè, non contente de se faire un nom, est devenue un monument.

Le récit que livre Paul Hazoumé prend la forme d'un témoignage : comme il le rappelle dans les premiers paragraphes, il aurait en effet recueilli dès 1917 les propos de l'amazone, dont le souvenir lui serait revenu en mémoire à l'annonce de sa mort en 1924. La biographie de Tata Ajachè constituerait donc, à en croire le dispositif mis en œuvre par Hazoumé, un témoignage de première main, d'autant plus difficile à recueillir que l'amazone aurait manifesté les plus grandes réserves et n'aurait accepté de raconter son histoire qu'après de longues heures d'approche, de nombreux présents - fagots de bois, jarres d'eau, discret flacon de cordial — et surtout après avoir obtenu la garantie que son auditeur ne prendrait aucune note. Seule l'excellente mémoire d'Hazoumé, dont l'éloge clôture d'ailleurs l'article, aurait par conséquent permis de conserver le récit de l'amazone avant qu'il ne se trouve tardivement consigné dans les pages de La Reconnaissance Africaine. On mesure dès lors ce que ce témoignage, fût-il étayé par une véritable rencontre, doit à la reconstruction a posteriori de l'auteur. Maurice Delafosse ne dit pas autre chose lorsqu'il loue, dans l'article qu'il donne à La Dépêche coloniale, l'hybridité générique du texte d'Hazoumé — hésitant entre le conte, l'anecdote et l'histoire :

Ce récit qui n'est pas un conte, est à la fois un très joli morceau littéraire, extrêmement vivant, et un modèle historique anecdotique, en même temps qu'il nous révèle des traits ignorés des mœurs guerrières et des coutumes de la cour des anciens rois d'Abomey.

Partagé entre la vocation testimoniale et la tentation feuilletoniste, l'article consacré à Tata Ajachè occupe une position intermédiaire entre la littérature et la documentation. Il semble avant tout destiné à valoriser l'apport de l'érudit local frotté de «régionalisme»: de fait, si Hazoumé parvient à

vieille serra les mâchoires, leva le bras droit, et, répétant le geste qu'elle avait fait avec sa houe, fit claquer le pouce contre le majeur. Une fureur bestiale passa fulgurante dans ses yeux) », in La Reconnaissance Africaine, n 1 , daté du 15 août 1925. 
recueillir et à transmettre le témoignage de l'amazone, c'est parce qu'il est doté d'une mémoire exercée par la tradition orale, et parce que sa connaissance des langues et des proverbes locaux lui permet de décoder bien mieux que ne le font les chercheurs européens les motifs symboliques constitutifs des bas-reliefs d'Abomey. En cela, les textes consacrés à l'amazone préparent l'éloge de l'érudit local que propose Hazoumé (1957) à l'occasion du Congrès des écrivains et artistes noirs de 1956. Centré sur un personnage féminin d'exception, le récit annonce cependant aussi le destin de l'héroïne éponyme de Doguicimi : contrairement à Ouidah et à Porto Novo, auxquels Hazoumé consacre des écrits beaucoup plus marqués par la préoccupation documentaire, le royaume impérialiste Abomey semble ainsi constituer pour lui le foyer d'une inspiration romanesque récurrente. Tata Ajachè apparaît à ce titre comme une préfiguration de Doguicimi amoureuse, avec qui elle partage une posture de loyauté absolue sous les tortures.

Ce personnage historique, intégré dans l'histoire monumentale du royaume d'Abomey, débouche ainsi sur l'invention concomitante de deux figures centrales du corpus hazouméen - celle de Doguicimi d'une part, héroïne fictive du roman historique à venir, et celle de l'érudit indigène d'autre part, habité par un humanisme bifide qui le rattache aussi bien à la tradition occidentale qu'aux cultures locales.

\section{Itinérances d'un espion}

L'intermédiaire particulier que constitue l'espion joue un rôle central dans l'intrigue de Doguicimi : c'est ainsi l'agent de renseignement Zanbounou qui prépare la campagne victorieuse menée pour libérer le prince Tofa, lorsque ce dernier tombe aux mains de l'ennemi lors des combats menés en pays mahi; c'est encore lui qui, par son faux témoignage, précipite la condamnation de Doguicimi et sa mise au supplice. L'importance accordée à ce personnage duplice justifie qu'on s'attache à son apparition précoce dans le troisième chapitre du Pacte de sang, publié d'abord dans les derniers numéros de La Reconnaissance Africaine.

L'examen de l'usage du pacte de sang par les espions autorise une inflexion dans l'économie générale de l'essai qui se détache, à l'occasion de l'évocation de cette figure politique et réaliste, du récit des origines légendaires de l'alliance. De fait, si le deuxième chapitre faisait la part belle au conte et insistait sur la dimension religieuse d'une pratique née du contact de l'homme avec les « génies ", le troisième chapitre lui confère une dimension sociologique que souligne la présence du mot « caste» dans le titre : au récit mythique des 
origines se substitue une interrogation du pacte comme support du lien social, voire comme instrument de conquête impériale. Le pacte de sang conclu entre l'espion et un dignitaire du pays ennemi constitue ainsi le premier pas préalable à toute opération militaire : il permet l'intégration de l'agent de renseignement à la cité adverse - voire son élection au rang de confident de l'ennemi. L'espion, dès lors, se place à la croisée des chemins entre la pratique rituelle et l'observation, entre le geste magique et la documentation. Le portrait de ce personnage duplice permet donc de ne pas reléguer le pacte de sang au rang des superstitions intemporelles, bercées d'un récit légendaire, et de mettre au contraire en évidence un usage politique de la tradition.

L'attache réaliste de l'espion est d'autant plus remarquable qu'elle va de pair avec une relative inconsistance du personnage, que son nécessaire anonymat condamne à échapper au regard de l'historien. La description de ce qu'Hazoumé présente comme un corps de métier spécialisé ne s'appuie que sur peu d'exemples, et l'auteur semble vouloir se disculper de cette parcimonie en insistant sur la difficulté qu'il y aurait à identifier des hommes contraints par leurs fonctions à la plus grande discrétion :

Ils étaient recrutés parmi toutes les peuplades, et devaient comprendre plusieurs dialectes. Les rois d'Agbomê avaient à leur service surtout des agbadjigbeto Mahi et Nago, qu'ils envoyaient de préférence dans leurs pays d'origine [...]. Dans un royaume, quelques hauts dignitaires seulement connaissaient les agbadjigbeto au service du pays. Leur nom et leur personne devaient être tenus cachés. $\mathrm{Si}$, malgré le mystère dont on entourait leur personnalité, quelques noms nous sont parvenus, il faut l'attribuer à la fréquence des missions effectuées par certains d'entre eux (Hazoumé 1937 : 20).

Les espions se présentent ainsi comme des figures structurellement inconnues, dont seul un hapax historique peut parfois permettre de proposer une identification : écrire, à travers le prisme offert par le pacte de sang, l'histoire de l'espionnage au royaume du Dahomey revient par conséquent à forger une histoire anonyme, privée des sources indispensables à l'historien. L'espion, en somme, se révèle presque aussi inaccessible que le génie Aziza dont le chapitre précédent rapportait les rares apparitions. On ajoutera enfin que l'espion est d'autant plus labile et insaisissable qu'il se présente comme une figure polyglotte, exilée, souvent elle-même issue d'un processus ancien de conquête qui la cantonne à une position subalterne éminemment précaire. De fait, le risque assumé par l'espion d'Abomey ne se limite pas au danger d'une découverte qui compromettrait son anonymat alors qu'il se trouve en terrain ennemi : bien plus, le péril que pointe Hazoumé se situe au moment du retour à Abomey, en cas d'échec de l'entreprise de conquête que la mission 
d'espionnage se devait de préparer. Il rappelle ainsi qu'à son retour, l'espion fait l'objet d'une séquestration systématique et se voit exposé à la peine de mort dans le cas où son action n'aurait pas suffi à tromper la vigilance de l'ennemi.

Le défaut d'informations précises et la situation ambiguë des espions semblent alimenter une empathie de l'écrivain envers ces personnages de l'entre-deux, partagés entre une double loyauté consacrée par la promesse et le rituel. Il est possible que le mystère qui entoure ces personnages ait facilité leur réappropriation romanesque, ou ait entretenu, comme nous souhaiterions le suggérer ici, la possibilité d'une identification de l'auteur à ces figures d'intermission problématique ${ }^{5}$. Plusieurs indices contribuent ainsi à étayer un parallèle entre le positionnement de l'auteur assimilé, tiraillé entre des influences et des impératifs contradictoires, et le double bind auquel se trouve soumis l'espion, tenu à la fois par l'allégeance qu'il a faite au roi et par le pacte de confiance mutuelle qu'il a conclu en pays ennemi. Le conflit est encore plus insoluble si, comme le suggère Hazoumé au début du chapitre, l'agent double a été envoyé en mission dans son pays d'origine qu'il se voit contraint de trahir. Le développement consacré aux espions s'achève ainsi sur l'évocation mélancolique d'un dilemme irrésolu :

Malgré ce bonheur, une certaine angoisse surgissait dans l'esprit de l'espion : Qu'étaient devenus ses «frères » au pays conquis ? avaient-ils péri dans la bataille ? Avaient-ils été capturés puis immolés à l'occasion de la fête qui, de coutume, suivait la victoire? Ils gémissaient peut-être dans les fers, en captivité ; leurs familles avaient-elles été massacrées ou capturées comme eux, leurs cases pillées, puis sans doute incendiées?

Et maintenant, lui, agbadjigbeto, auteur de tous ces maux, échapperait-il aux terribles châtiments réservés au parjure? (Hazoumé 1937 : 26).

Cette clôture du chapitre marque une nette rupture par rapport au ton objectif et scientifique qui caractérisait les paragraphes précédents. Hazoumé abandonne le point de vue surplombant de l'historien pour adopter celui de l'espion assailli par le doute au lendemain de la victoire : ce faisant, il refuse le prisme exclusif d'une histoire écrite « par les vainqueurs » (de Certeau 2002),

5. Cette identification est d'autant plus saisissante que l'ironie de l'histoire (post)coloniale a fait d'Antoine Hazoumé, fils de Paul Hazoumé, un agent des renseignements français, vraisemblablement recruté dans le courant des années 1960 par le Service de documentation extérieure et de contre-espionnage. Un témoignage littéraire et intime de cette période est livré par Flore Hazoumé, fille d'Antoine Hazoumé, dans la collection des «Classiques ivoiriens » (HAZOUMÉ 2016). 
et critique au passage la violence d'un royaume impérialiste qui met à feu et à sang les campagnes conquises. Plus encore, il s'approprie dans ces dernières lignes une figure dont l'insuffisance de la documentation historique renforce la disponibilité narrative. Si le chapitre des espions se clôt sur une incertitude, il ne s'agit plus en effet du flou des sources que soulignait Hazoumé à l'orée de sa réflexion, mais d'une véritable interrogation éthique et identitaire. Le bref monologue de l'espion reflète l'impossible positionnement de celui qui, appartenant à deux cultures ou à deux sociétés rivales, est condamné à la trahison et refuse de s'en accommoder : l'inquiétude que véhicule la question rhétorique finale ne saurait donc être réduite à la seule crainte d'une rétribution magique qui s'exprimerait à travers l'une des «sanctions » que détaille le cinquième chapitre de l'essai. Le doute qui étreint l'espion se lit tout autant comme l'inquiétude chrétienne d'une conscience tourmentée, ou comme le travail douloureux d'une identité partagée. À ce titre, il ne nous semble pas anodin que la dernière phrase opte précisément, pour évoquer l'espion fauteur de troubles et agent d'une désorganisation de la cité, pour une métaphore auctoriale, en le désignant comme l' « auteur de tous ces maux ». Il n'est que trop tentant de lire la poussée d'empathie à laquelle donne voix Hazoumé comme la manifestation d'une identification de l'écrivain à la figure creuse et historiquement inoccupée de l'espion dahoméen. L'auteur ne fonde-t-il pas sa position, comme l'espion, sur une connaissance des langues locales, dont il se fait, dès ses premiers articles, l'interprète pour l'Occident? Ne se livret-il pas, comme l'espion encore, à un travail d'observation et de recension de son pays natal, au point de se voir accusé d'en avoir trahi les secrets et les savoirs ésotériques? Ne revendique-t-il pas enfin dans l'introduction le souci de constituer une documentation qui appuie l'entreprise coloniale française? Les parallèles sont assez nombreux pour autoriser l'hypothèse d'une projection d'Hazoumé dans la figure dystopique de l'espion à la conscience malheureuse, justifiant ainsi le changement de ton brutal qui suspend le chapitre dans une indécision douloureuse.

\section{Pour une poétique de la nostalgie}

Si l'amazone Tata Ajachè prépare l'avènement de la figure héroïque que constitue Doguicimi, son interlocuteur retors, prompt à ruser pour obtenir d'elle l'information qu'il souhaite, trace le portrait ambigu d'un ethnographe local, proche à bien des égards de l'espion en mission. Cette figure intermédiaire complexe est vouée à connaître de nombreuses apparitions dans les écrits d'Hazoumé et donne lieu au déploiement, au cœur même de l'essai 
ethnographique, d'un discours souvent subjectif, ou à tout le moins irréductible à sa seule teneur informative. La mélancolie dont il se révèle porteur participe plus largement, sous la plume de Paul Hazoumé, d'une évocation nostalgique du Dahomey précolonial. Son décor privilégié est celui des ruines, dont le motif était déjà particulièrement prisé par les auteurs romantiques et orientalistes (Mortier 1974). Là encore, l'attention accordée à cette topique conduit à intégrer les textes hazouméens dans un continuum entre documentation et littérature, tout en suggérant l'insuffisance d'une assignation sans nuance de l'auteur au rang des écrivains colonialistes.

\section{Le mensonge du chef-féticheur}

Largement tributaire de l'influence de Francis Aupiais, Le Pacte de sang au Dahomey accorde une place prépondérante aux considérations portant sur la spiritualité indigène, particulièrement présentes à l'orée et à la fin du texte. S'ouvrant sur un hommage appuyé à Aupiais et à l'œuvre civilisatrice des missionnaires, l'essai est clos par l'évocation de la visite d'un temple vodoun, traditionnellement entouré du plus grand mystère. La description inédite du lieu de culte que propose à cette occasion Paul Hazoumé, fidèle à la posture de l'intermédiaire introduit, n'est cependant pas exempte d'ambiguïté et ne saurait passer pour la seule manifestation d'un prosélytisme chrétien. L'évocation du temple oscille en effet entre la condamnation de pratiques païennes vouées à la disparition et l'évocation nostalgique d'un passé révolu:

Ce jour-là, le chef-féticheur était absent. Nous avons pu pénétrer dans le bosquet d'Oguêrê, grâce à la complicité de notre cicérone. Après avoir traversé les vestiges des neuf portes qui restaient sur les seize du temps de la toute-puissance du vodoun, nous arrivâmes à un emplacement circulaire qui pouvait bien mesurer deux mètres cinquante de diamètre. Le chemin qui conduit dans l'enceinte était jonché d'une épaisse couche de feuilles sèches que perçaient çà et là des herbes folles. Tout disait que depuis des lunes, aucun pied humain n'avait foulé ces lieux.

Au fond de l'enceinte, deux jeunes kolatiers se dressaient côte à côte. À leurs pieds étaient entassés des tessons de bouteille, des débris de poterie du pays et de vaisselle européenne. Un cruchon au col ébréché était à demi renversé face aux kolatiers. C'était sans doute celui qui servait à puiser l'eau d'offrande dans le Hinto à l'époque des cérémonies dont le souvenir est encore présent dans les esprits.

Nous avons exploré à loisir le bosquet et n'y avons rien découvert de sensationnel (Hazoumé 1937 : 167). 
Hazoumé se place ici non seulement dans une position de transmission médiée, recourant à l'indispensable truchement d'un guide qualifié de " cicérone », mais encore dans une situation de transgression. Il ne pénètre en effet au sein du bosquet sacré qu'en profitant de l'absence ponctuelle du chef-féticheur, dont une note rapporte qu'il est également le chef du village et s'est éclipsé pour participer à une réunion consacrée au percement d'une route. Cette incursion clandestine de l'ethnographe est d'autant plus sacrilège qu'elle permet de dévoiler, dans les dernières lignes de l'essai, le mensonge du féticheur hâbleur qui assure à son interlocuteur qu'il a récemment encore obtenu la guérison d'un malade.

Le chef féticheur que nous avons rejoint quelques moments après, nous parla longuement des prodiges du vodoun Oguêrê. À notre question : «Les possédés viennent-ils toujours chercher la guérison à Dô ? », il répondit incontinent d'un ton plein d'assurance : «il y a deux marchés à peine, j'ai imploré Oguêrê pour un parjure expiant ses fautes et j'ai obtenu la guérison du malheureux. Je suis prêt à prier le vodoun pour tous les malades qu'on m'amènera. »

Rompant le pacte de confiance sous-jacent à l'entretien ethnographique, la parole du sujet interrogé se présente d'emblée comme un faux témoignage que l'expérience de terrain de l'enquêteur contrevenant permet d'invalider : le lieu de culte est déserté, privé de ses fidèles aussi bien que de son serviteur appelé à d'autres fonctions. La déclaration du féticheur qui clôt l'essai — sans qu'Hazoumé juge même nécessaire, au regard des lignes qui précèdent, de lui infliger un démenti formel - apparait par conséquent comme un ultime acte de déni ou comme un vœu pieux flottant sans objet sur les vestiges d'une religion privée d'adeptes. Il n'est que trop aisé de lire dans cette représentation le triomphe en creux de la religion chrétienne : les derniers paragraphes du texte feraient alors écho à la lettre dédicatoire en témoignant de l'effacement des pratiques animistes au profit de la diffusion de la foi chrétienne. La conversion du féticheur lui-même, devenu chef de village et chargé de participer à l'établissement du tracé des routes, confirme la pénétration de la " civilisation » européenne dans les structures politiques et religieuses locales.

Pourtant, cette représentation du temple comme ruine semble autoriser, par l'absence de commentaire ou de formule de clôture, plusieurs lectures concurrentes ou complémentaires : en effet, si l'édifice en ruines est décrit comme un lieu de déréliction, où les objets du culte se sont transformés en «tessons » et en « débris », il n'en revêt pas moins une certaine majesté silencieuse, caractéristique des poétiques de la ruine. Le temple que découvre Hazoumé, quoique gagné par une nature conquérante, est toujours habité par 
les « souvenirs » d'une pratique ancienne : il s'écarte certes du «sensationnel » prisé dans les chroniques de voyageurs en quête d'exotisme, mais il n'en aspire pas moins à une forme de sublime dont la ruine est l'expression privilégiée, en tant qu'elle véhicule selon Adorno (1978) une « mémoire de la transcendance ». La description aboutit donc à une double lecture du monument, perçu à la fois comme le reliquat délabré d'une pratique révolue et comme un lieu de mémoire où trouvent à s'exprimer les derniers échos d'une grandeur déchue. Loin de porter une condamnation sans appel du vodoun, le propos semble par conséquent l'inscrire dans la continuité historique d'un chemin vers la transcendance. Plus encore, la clôture de l'essai, qui s'achève avec la prise de parole du chef-féticheur, émancipée de tout commentaire de l'auteur, n'est elle-même pas dépourvue d'ambiguité. Cette déclaration se comprend en effet aussi bien comme un mensonge - symptôme de la mauvaise foi inhérente à la croyance païenne et de son caractère illusoire - que comme un acte de piété : au vestige du passé que constitue la ruine ferait alors pendant le futur hypothétique d'une irréductible profession de foi.

Si Le Pacte de sang se place explicitement sous un patronage chrétien - au point de susciter les réticences de l'Institut d'ethnographie — sa clôture ne coïncide donc pas avec la condamnation des pratiques du vodoun que laissait supposer le portrait liminaire d'une fillette martyrisée, sauvée in extremis par l'intervention opportune des missionnaires. La représentation du temple en ruines suscite plutôt un regard historique teinté de mélancolie et un discours dont le dernier mot, fût-il mensonger, revient toujours au féticheur.

\section{Ruines impériales, vestiges familiaux}

Le « Journal de voyage de Cotonou à Dassa-Zoumé $\rangle^{6}$ reste un texte exceptionnel dans l'entreprise « régionaliste» de La Reconnaissance Africaine : difficilement rattachable aux «études historiques 》 ou à l'apologétique chrétienne, il se rapproche bien plus du récit de voyage ou de l'autobiographie indigène. L'entreprise pourrait à ce titre être assimilée à ce que Mary-Louise Pratt (1992) appelle une « autoethnographie », susceptible d'entrer en dialogue avec les discours tenus en métropole sur les sujets colonisés. Le texte permet en premier lieu d'éclairer un versant méconnu de la biographie d'Hazoumé, en rappelant que sa mère aurait été faite prisonnière par les troupes du royaume d'Abomey:

6. Voir La Reconnaissance Africaine, $\mathrm{n}^{\mathrm{o}} 13$ (15/03/1926), $\mathrm{n}^{\circ} 14(01 / 04 / 1926), \mathrm{n}^{\circ} 16$ (01/05/1926), no 19 (15/06/1926), no 20 (01/07/1926), no $22(01 / 08 / 1926), n^{\circ} 27$ $(15 / 10 / 1926), n^{\circ} 29(15 / 11 / 1926)$. 
Je suis venu presque sans but dans ce pays, cependant je ne puis ne pas profiter des trois jours que nous y resterons sans chercher à revoir des parents que je sais exister dans les montagnes voisines, un oncle en particulier — qui n'a pas revu sa sœur, ma mère — depuis l'époque où celle-ci — encore enfant — avait été ravie par l'armée dahoméenne au cours d'un pillage (La Reconnaissance Africaine, $\mathrm{n}^{\circ} 13$ ).

Le détour autobiographique met donc en évidence une généalogie qui fait d'Hazoumé, non seulement l'héritier d'une tradition royale autonome et rivale issue de Porto-Novo, mais aussi le descendant d'une victime de la politique impérialiste du royaume d'Abomey. Le voyage vers le Nord se transforme dès lors en pèlerinage sur les lieux d'un rapt, dont Hazoumé partage 1'histoire avec ses personnages féminins, Doguicimi et l'amazone Tata Ajachè.

Avant de retracer ce retour au pays natal, le récit s'ouvre sur une description du voyage en train, qui incite l'auteur à souligner la variété des langues et des peuples qui constituent le Dahomey au-delà de la zone d'influence d'Abomey. Puis, alors qu'il se rapproche de sa destination, à proximité de la gare de Dan, Hazoumé est invité par un compagnon de voyage à contempler depuis l'intérieur du wagon les ruines témoins de la chute de l'ancien royaume :

Mon guide me signale à notre droite à quelques pas de la voie, des ruines au milieu desquelles s'élève un superbe fromager : voici nous dit-il, le lieu de retraite du roi Béhanzin après son départ précipité d'Abomey à l'approche de l'armée du " Généla ». Ces ruines envahies par les herbes folles manquent de grandeur et même de tristesse ! Cependant elles ont assisté à la chute d'une puissance qui se croyait invincible. Que de messages de jour et de nuit ont dû venir à ce village, apportant chacun de leur voyage, mêlée de fausses bonnes nouvelles, la certitude de plus en plus avérée que le royaume d'Abomey était bien détruit (La Reconnaissance Africaine, $\mathrm{n}^{\circ} 14$ ).

Les points de rencontre entre ce texte et la description du temple abandonné sont nombreux, tenant aussi bien à la présence d'un guide qu'au triomphe de la végétation et des « herbes folles » qui poussent entre les pierres et scellent le déclin d'une civilisation défaite. Le feuilleton paru dans les pages de $L a$ Reconnaissance Africaine, en évoquant le compte à rebours inexorable d'une défaite rythmée par l'arrivée de trompeurs coursiers de Marathon, exprime néanmoins plus clairement encore la conscience d'une grandeur révolue et de la faillibilité des puissances séculières.

Le déploiement d'une poétique de la ruine ne s'arrête cependant pas à cette seule mention ponctuelle: de fait, si les retrouvailles escomptées avec l'oncle maternel ont bien lieu, le voyage en pays dassa reste une expérience 
largement déceptive - au point que le récit n'est pas mené à son terme et s'interrompt brutalement, avant que l'ancêtre n'ait eu le temps d'évoquer les exactions commises par les troupes d'Abomey. D'emblée, à Dassa-Zoumé, l'entrevue d'Hazoumé avec sa tante, qu'il sollicite pour trouver un guide, se solde par le constat de son aliénation : lorsqu'il envisage de se faire transporter en hamac, l'aïeule ne manque pas de lui reprocher amèrement son éducation européenne et son infidélité aux valeurs familiales. Pour ne pas être perçu comme un produit timoré de l'assimilation, Hazoumé s'engage donc à parcourir la route à pied: la difficulté du trajet en fait une véritable épreuve initiatique, sans pour autant qu'elle débouche sur une rencontre ou une révélation qui permettrait la récupération de l'identité perdue ${ }^{7}$. L'itinéraire qui mène le narrateur au village où il cherche son oncle lui paraît dangereux et semé d'embûches. Soupçonnant à tort ses deux guides — qui se sont pourtant présentés à lui comme des cousins — d'en vouloir à sa bourse et à sa vie, il se révèle incapable de renouer avec les structures sociales et familiales du village maternel. La fête qu'on lui fait à son arrivée ne suffit pas à révoquer ce climat de méfiance et de communication entravée, qui fait d'Hazoumé un étranger d'autant plus paradoxal qu'on s'étonne de l'entendre maîtriser la langue dassa. L'écrivain assimilé devient ainsi un errant paranoïaque, balloté par ses accompagnateurs de cases en ruines, de crêtes en collines: sa quête semble sans fin, puisque la rencontre escomptée avec l'oncle aboutit à l'entreprise d'un nouveau pèlerinage dans la montagne. Lorsque le narrateur parvient enfin, au terme de longs détours, sur les vestiges de la case maternelle, le récit s'interrompt brutalement sur une promesse de suite que le bulletin, on s'en souvient, ne tient pas. C'est donc de nouveau sur une description de ruines que s'achève ce récit de voyage :

Puis le vieillard, tendant son bâton vers un groupe de cases, me dit : « Tu es dans le quartier de tes parents. » À l'emplacement où existait le village, deux ans auparavant, il n'y a plus guère que des ruines mais ici l'herbe ne dépasse pas les genoux. Quelques pas nous amènent devant les cases de mes parents, encore couvertes de leur toit déjà bien affaissé. De la plupart des cases environnantes les murs sont presque au ras du sol. Encore trois ou quatre saisons de pluie et tout sera nivelé et caché sous la brousse, à la grande joie de la gent animale qui remplacera les hommes en ces lieux (La Reconnaissance Africaine, $\mathrm{n}^{\circ} 29$ ).

Refusant toute forme de conclusion ou de discours mémoriel, le récit du retour au pays natal n'aboutit ainsi qu'à la contemplation nostalgique de

7. Nous nous référons ici à la définition du récit initiatique proposée par X. GARNIER (2004: 452) : «Le récit initiatique n'est pas la relation d'une rencontre mais sa mise en œuvre. Toutes les composantes du récit sont mobilisées par la rencontre, elles en sont l'effectuation. La figure itinérante n'est pas un personnage, mais un "être-pour-la-rencontre". » 
la ruine et au constat de l'inéluctable disparition du souvenir des vaincus, recouvert par une nature envahissante.

Le Dahomey qui transparaît dans l'œuvre d'Hazoumé est une terre de ruines et de monuments - monuments anciens comme ceux de la cour du palais d'Abomey qui commémorent, entre autres, les exploits sanglants de Tata Ajachè, monuments récents comme celui auquel s'adosse le projet de La Reconnaissance Africaine. Il ne saurait dès lors être compris uniquement comme un Dahomey « documentaire », conçu pour alimenter la connaissance coloniale du terrain à administrer : il est aussi un Dahomey « régional», dont les élites s'attachent à concilier les « deux humanismes » de l'Occident et de l'Afrique (Hazoumé 1957), et un Dahomey «historique», dont les textes s'emploient à construire et à transmettre la mémoire lacunaire. Franchir «la porte de l'école» revient par conséquent pour l'écrivain francophone à prêter attention à une architecture nouvelle, faite d'estompements et de superpositions. Si Hazoumé accorde un indubitable primat au local qu'il inventorie, il n'en oscille pas moins entre deux mondes, deux loyautés et deux impérialismes rivaux. Le Pacte de sang et les textes parus dans La Reconnaissance Africaine portent les prémisses des constructions romanesques à venir et recèlent une esthétique propre qui témoigne d'un investissement subjectif du document scientifique. L'inscription des écrits ethnographiques de Paul Hazoumé dans un continuum souple allant du document à la littérature permet ainsi l'identification d'une poétique de la ruine qui apparaît comme le pendant des errements identitaires de l'écrivain assimilé et de l'érudit régionaliste. Selon Emmanuel Hocquart (2010 : 11-12), le motif littéraire de la ruine recèle une véritable fonction expressive dans la mesure où il désigne «la tombée de ce qui, auparavant, servait à séparer ou à faire communiquer, sur une infinité de modes, des dedans et des dehors » : de même, la ruine hazouméenne apparaît moins comme un décombre que comme une rupture de toutes les cloisons - jusqu'à celles qui auraient pu articuler l'identité d'un écrivain devenu suspect d'espionnage.

Théorie et histoire des arts et des littératures de la modernité (THALIM), Université Paris-3-Sorbonne Nouvelle, Paris. 


\section{BIBLIOGRAPHIE}

Adorno T., 1978, Dialectique négative, Paris, Payot.

Aggarwal K., 1999, Amadou Hampâté Bâ et l'africanisme: de la recherche anthropologique à l'exercice de la fonction auctoriale, Paris, L'Harmattan.

Anderson B., 1996, L'imaginaire national. Réflexions sur l'origine et l'essor du nationalisme, Paris, La Découverte.

AupIAIs F., 1927, «Une tentative de régionalisme africain », in COLLECTIF (dir.), Les élites en pays de mission : compte-rendu de la cinquième semaine de missiologie de Louvain (1927), Louvain, Éditions du Museum Lessianum : 90-100.

— 2016, Cours et causeries pour « réhabiliter les Noirs », Rome, SMA.

- 2018, Lettres à Paul Hazoumé, Rome, SMA Publications («SMA Sankofa, 12), éd. P. Saulnier.

BALARD M., 1999, Dahomey 1930 : mission catholique et culte vodoun. L'œuvre de Francis Aupiais (1877-1945), missionnaire et ethnographe, Paris, L'Harmattan.

BÉNICHOU P., 1996, Le Sacre de l'écrivain : 1750-1830. Essai sur l'avènement d'un pouvoir spirituel lä̈qu dans la France moderne, Paris, Gallimard.

Biton M.-M., 2000, L'Art des bas-reliefs d'Abomey, Paris, L'Harmattan.

De Certeau M., 2002 [1975], L'Écriture de l'histoire, Paris, Gallimard.

Coundouriotis E., 1999, Claiming History: Colonialism, Ethnography, and the Novel, New York, Columbia University Press.

DeBAENE V., 2010, L'Adieu au voyage : l'ethnologie française entre science et littérature, Paris, Gallimard.

DERIVE J., 1980, « Littérature négro-africaine et critique universitaire : identité d'un objet et spécificité des méthodes », Ethnopsychologie, 2-3 : 41-59.

Dubreuil L., 2008, L'Empire du langage : colonies et francophonie, Paris, Hermann.

Ducournau C., 2017, La fabrique des classiques africains : écrivains d'Afrique subsaharienne francophone, 1960-2012, Paris, CNRS éditions.

GANDONOU A., 2002, Le roman ouest-africain de langue française : étude de langue et de style, Paris, Karthala.

Garnier X., 2004, «À quoi reconnaît-on un récit initiatique?», Poétique, 140 (4) : 443-454. 
Gary-Tounkara D. \& Nativel D., 2012, "Questionner la fabrique des savoirs », in D. Gary-TounKara \& D. Nativel (dir.), L'Afrique des savoirs au sud du Sahara (XVI $-X X I^{e}$ siècle). Acteurs, supports, pratiques, Paris, Karthala : 10-25.

HARDy G., 1949, Un apôtre d'aujourd'hui: le Révérend Père Aupiais, provincial des Missions africaines de Lyon, Paris, Larose.

Hazoumé F., 2012, Je te le devais bien, Abidjan, Les Classiques Ivoiriens.

Hazoumé P., 1937, Le Pacte de sang au Dahomey, Paris, Institut d'ethnologie.

— 1938, Doguicimi, Paris, Maisonneuve et Larose (deuxième édition en 1972).

— 1957, «L'humanisme occidental et l'humanisme africain », Présence africaine, XIV-XV : 29-45.

Hocquart E., 2010, Ruines à rebours, Bordeaux, Éditions de l'attente.

Huannou A. \& Mane R. (dir.), 1987, Doguicimi de Paul Hazoumé, Paris, L'Harmattan.

JezeQuel J.-H., 2007, « Voices of their Own? African Participation in the Production of Colonial Knowledge in French West Africa, 1889-1919», in H. TILlEY \& R. Gordon (eds.), Ordering Africa : Anthropology, European Imperialism, and the Politics of Knowledge, Manchester, Manchester University Press : 145-172.

KInG A., 2002, Rereading Camara Laye, Lincoln, University of Nebraska Press.

Labrune-Badiane C. \& SMith É., 2018, Les Hussards noirs de la colonie. Instituteurs africains et «petites patries » en AOF (1913-1960), Paris, Karthala.

LAWrance B., Osborn E. \& Roberts R. (eds.), 2006, Intermediaries, Interpreters and Clerks : African Employees in the Making of Colonial Africa, Madison, The University of Wisconsin Press.

MorTIER R., 1974, La Poétique des ruines en France: ses origines, ses variations de la Renaissance à Victor Hugo, Genève, Droz.

PRATT M.-L., 1992, Imperial Eyes : Travel Writing and Transculturation, London-New York, Routledge.

RIESZ J., 2007, « De l'ethnographie à la naissance du roman africain », in De la littérature coloniale à la littérature africaine, Paris, Karthala : 249-268.

Senghor L. S., 1947, Les plus beaux écrits de l'Union Française et du Maghreb, Paris, La Colombe.

WARnER T., 2016, «Para-literary Ethnography and Colonial Self-writing: The Student Notebooks of the William Ponty School », Research in African Literatures, 47 (1): 1-20. 
RÉSUMÉ

En proposant une analyse des travaux ethnographiques de l'écrivain dahoméen Paul Hazoumé, cet article établit un continuum entre littérature et science. Alors même que Doguicimi, unique roman historique de l'auteur, est souvent réduit au statut de "document» ethnographique, nous proposons d'inverser la perspective en relisant ses écrits scientifiques - Le Pacte de Sang au Dahomey et les articles parus dans la revue régionaliste du père Francis Aupiais, La Reconnaissance Africaine — en adoptant un prisme littéraire. Le présent article met ainsi en évidence la construction de «personnages ethnographiques », susceptibles de devenir le support d'un récit ou d'un discours littéraire, et examine l'émergence d'une poétique de la ruine propice à la description du Dahomey colonial.

Mots-clés: Dahomey, auctorialité, informateur colonial, littérature francophone, paysage, religion, régionalisme

\section{ABSTRACT}

"Ruines à rebours". For a Literary Reading of Paul Hazoumé's Ethnographic Writings. By analysing the ethnographical work of the Dahomean writer Paul Hazoumé, this article aims at establishing a continuum between literature and science. Whereas Hazoumé's historical novel Doguicimi has often been perceived as a clumsy attempt at fiction by a colonial informer, we propose to read the essay Le Pacte de sang au Dahomey and the articles published in La Reconnaissance africaine, a local journal created by the missionary Francis Aupiais, as narrative and poetic experiments. Hazoumé's first writings allow the emergence of "ethnographical characters" such as the spy and the female warrior, and they suggest an aesthetic of ruins which would enable a nostalgic depiction of colonial Dahomey.

Keywords: Dahomey, colonial informer, francophone literature, intermediaries, landscape, regionalism, religion 